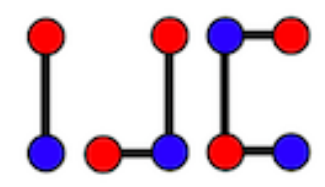

\title{
Four new operations related to composition and their reformulated Zagreb Index
}

\author{
K. Pattabiraman ${ }^{\mathrm{a}}$, A. Santhakumar ${ }^{\mathrm{b}}$ \\ ${ }^{a}$ Department of Mathematics, Annamalai University, Annamalainagar 608002 , India \\ ${ }^{b}$ Department of Mathematics, CK College of Engineering and Technology, Cuddalore, India \\ pramank@gmail.com, Santha.santhasulo.kumar8@gmail.com
}

\begin{abstract}
The first reformulated Zagreb index $E M_{1}(G)$ of a simple graph $G$ is defined as the sum of the terms $\left(d_{u}+d_{v}-2\right)^{2}$ over all edges $u v$ of $G$. In 2017, Sarala et al. [3] introduced four new operations $(F$ product) of graphs. In this paper, we study the first reformulated Zagreb index for the $F$-product of some special well-known graphs such as subdivision and total graph.
\end{abstract}

Keywords: Reformulated Zagreb index, composition, subdivision, total graph.

Mathematics Subject Classification : 05C12, $05 \mathrm{C} 76$

DOI: 10.19184/ijc.2018.2.1.5

\section{Introduction}

For vertex $u \in V(G)$, the degree of the vertex $u$ in $G$, denoted by $d_{G}(u)$, is the number of edges incident with $u$ in $G$. A topological index of a graph is a parameter related to the graph; it does not depend on labeling or pictorial representation of the graph. In theoretical chemistry, molecular structure descriptors (also called topological indices) are used for modeling physicochemical, pharmacologic, toxicologic, biological and other properties of chemical compounds [5]. Several types of such indices exist, especially those based on vertex and edge distances. One of the most intensively studied topological indices is the Wiener index. Two of these topological indices are known under various names, the most commonly used ones are the first and second Zagreb indices.

The Zagreb indices have been introduced more than thirty years ago by Gutman and Trinajestic [6]. They are defined as $M_{1}(G)=\sum_{u \in V(G)} d_{G}(u)^{2}$ and $M_{2}(G)=\sum_{u v \in E(G)} d_{G}(u) d_{G}(v)$. Note that the

Received: 30 Aug 2017, Revised: 29 Sep 2017, Accepted: 01 May 2018. 
first Zagreb index may also written as $M_{1}(G)=\sum_{u v \in E(G)}\left(d_{G}(u)+d_{G}(v)\right)$. The Zagreb indices are found to have appilications in QSPR and QSAR studies as well, see [4]. For the survey on theory and application of Zagreb indices see [9]. Feng et al.[7] have given a sharp bounds for the Zagreb indices of graphs with a given matching number. Khalifeh et al. [8] have obtained the Zagreb indices of the Cartesian product, composition, join, disjunction, and symmetric difference of graphs.

Furtula and Gutman in [16] recently investigated this index and named this index as forgotten topological index or F-index and showed that the predictive ability of this index is almost similar to that of first Zagreb index and for the entropy and acetic factor, both of them yield correlation coefficients greater than 0.95 . The $F$-index of a graph $G$ is defined as $F(G)=\sum_{u \in V(G)} d_{G}^{3}(u)=$ $\sum_{u v \in E(G)}\left(d_{G}^{2}(u)+d_{G}^{2}(v)\right)$.

Recently, Shirdel et al.[15] introduced a variant of the first Zagreb index called hyper-Zagreb index. The hyper-Zagreb index of $G$ is denoted by $H M(G)$ and defined as $H M(G)=\sum_{u v \in E(G)}(d(u)+$ $d(v))^{2}$. In [15], the hyper-Zagreb indices of the Cartesian product, composition, join and disjunction of graphs are obtained. The hyper Zagreb indices of some classes of chemical graphs are obtained in $[11,13,14]$. Pattabiraman and Vijayaragavan have obtained the hyper-Zagreb indices of some special classes of graphs[20]. Some upper and lower bounds on hyper-Zagreb index for a connected graph are obtained by Falahati-Nezhad and Azari[19].

Milicević et al. [23] in 2004 reformulated the Zagreb indices in terms of edge-degrees instead of vertex-degrees $E M_{1}(G)=\sum_{e \in E(G)} d(e)^{2}$, where $d(e)$ denotes the degree of the edge $e$ in $G$, which is defined by $d(e)=d(u)+d(v)-2$ with $e=u v$. The use of these descriptors in QSPR study was also discussed in their report [23]. Reformulated Zagreb index, particularly its upper/lower bounds has attracted recently theat tention of many mathematicians and computer scientists, see $[21,22,23,24,25]$. In this paper, we study the first reformulated Zagreb index for the $F$-product of some special well-known graphs such as subdivision and total graph.

\section{Main Results}

The Cartesian product, $G \square H$, of the graphs $G$ and $H$ has the vertex set $V(G \square H)=V(G) \times$ $V(H)$ and $(u, x)(v, y)$ is an edge of $G \square H$ if $u=v$ and $x y \in E(H)$ or $u v \in E(G)$ and $x=y$. To each vertex $u \in V(G)$, there is an isomorphic copy of $H$ in $G \square H$ and to each vertex $v \in V(H)$, there is an isomorphic copy of $G$ in $G \square H$. The composition of two graphs $G$ and $H$ is denoted by $G[H]$. The vertex set of $G[H]$ is $V(G) \times V(H)$ and any two vertices $\left(u_{i}, v_{r}\right)$ and $\left(u_{k}, v_{s}\right)$ are adjacent if and only if $u_{i} u_{k} \in E(G)$ or $\left[u_{i}=u_{k}\right.$ and $\left.v_{r} v_{s} \in E(H)\right]$.

For a connected graph $G$, there are four related graphs as follows:

(i) The subdivision graph $S(G)$ is the graph obtained from $G$ by replacing each edge of $G$ by a path of length two.

(ii) $R(G)$ is obtained from $G$ by adding a new vertex corresponding to each edge of $G$, then joining each new vertex to the end vertices of the corresponding edge. 
(iii) $Q(G)$ is obtained from $G$ by inserting a new vertex into each edge of $G$, then joining with edges those pairs of new vertices on adjacent edges of $G$.

(iv) The total graph $T(G)$ has as its vertices the edges and vertices of $G$. Adjacency in $T(G)$ is defined as adjacency or incidence for the corresponding elements of $G$, see Figure 1 .
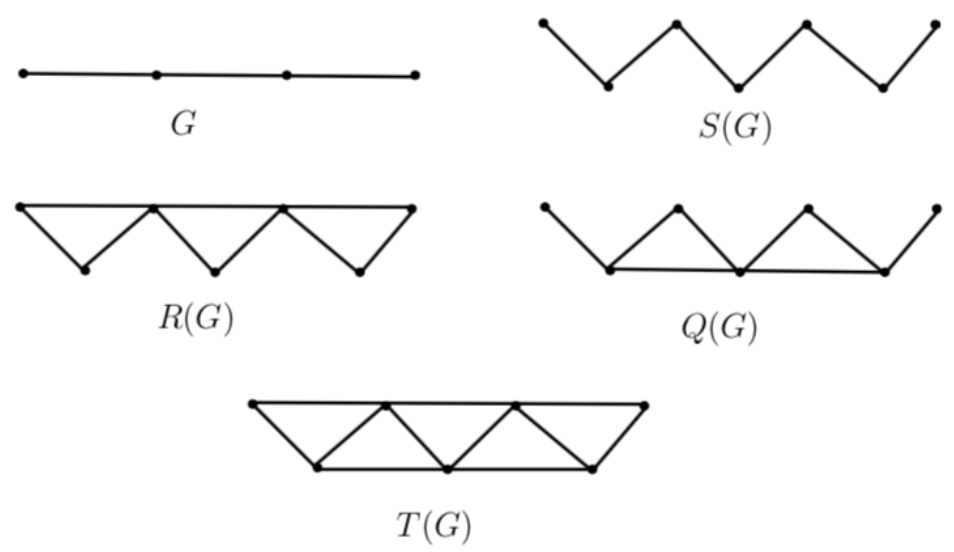

Figure 1. The graph $G$ its $S(G), R(G), Q(G)$, and $S(G)$.

Eliasi and Taeri [2] introduced the following four operations of the graphs $G_{1}$ and $G_{2}$ based on the Cartesian product of these graphs.

Let $F$ be one of the symbols $S, R, Q$, or $T$. The $F$-sum $G+{ }_{F} H$ is a graph with the set of vertices $V\left(G+{ }_{F} H\right)=(V(G) \cup E(G)) \times V(H)$ and two vertices $\left(g_{1}, h_{1}\right)$ and $\left(g_{2}, h_{2}\right)$ of $G+_{F} H$ are adjacent if and only if $g_{1}=g_{2}$ and $h_{1} h_{2} \in E(H)$ or $h_{1}=h_{2}$ and $g_{1} g_{2} \in F(G)$, see Figure 2. The Zagreb indices of the $F$-sum of graphs are obtained by Deng et al. [17]. The $F$-index of four operations on some special graphs are computed by Ghobadi and Ghorbaninejad [18]. Eliasi and Taeri[2] have obtained the Wiener index of four new sums of graphs.

In this sequence, Sarala et al. [3] introduced the following four operations of the graphs $G_{1}$ and $G_{2}$ based on the composition of these graphs.

Let $F$ be one of the symbols $S, R, Q$ or $T$. The $F$-product of $G_{1}$ and $G_{2}$, denoted by $G_{1}\left[G_{2}\right]_{F}$ is defined by $F\left(G_{1}\right)\left[G_{2}\right]-E^{*}$, where $E^{*}=\left\{\left(x, y_{1}\right)\left(x, y_{2}\right) \in E\left(F\left(G_{1}\right)\left[G_{2}\right]\right) \mid x \in V\left(F\left(G_{1}\right)\right)\right.$ $\left.V\left(G_{1}\right), y_{1} y_{2} \in E\left(G_{2}\right)\right\}$, that is, $G_{1}\left[G_{2}\right]_{F}$ is a graph with the set of vertices either $\left[x_{1}=x_{2} \in\right.$ $V\left(G_{1}\right)$ and $\left.y_{1} y_{2} \in E\left(G_{2}\right)\right]$ or $\left[x_{1} x_{2} \in E\left(G_{1}\right)\right.$ and $\left.y_{1}, y_{2} \in V\left(G_{2}\right)\right]$, see Figure 3. Sarala et al. [3] have obtained the Zagreb indices of $F$-product of graphs. 
Four new operations related to composition and ...
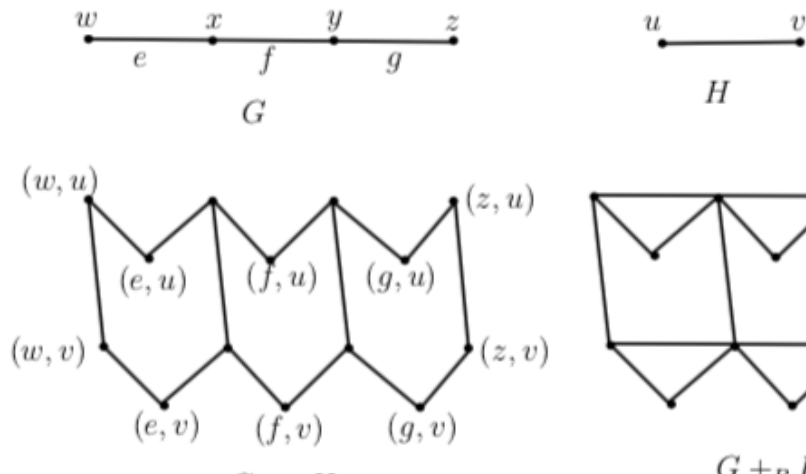

$G+{ }_{S} H$

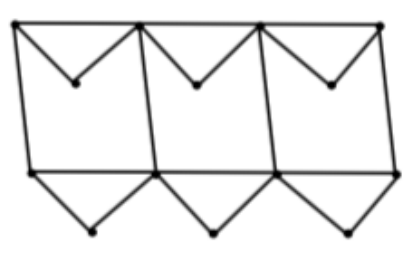

$G+_{R} H$
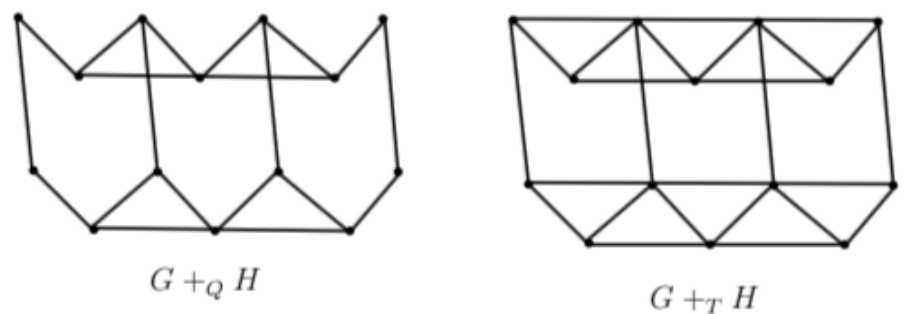

Figure 2. The graph $G, H$, and its $G+{ }_{F} H$.

First we compute the first reformulated Zagreb index of the graph $G_{1}\left[G_{2}\right]_{S}$.

Theorem 2.1. Let $G_{i}$ be a graph with $n_{i}$ vertices and $m_{i}$ edges, $i=1,2$. Then $E M_{1}\left(G_{1}\left[G_{2}\right]_{S}\right)=n_{1} H M\left(G_{2}\right)+n_{2}^{4} F\left(G_{1}\right)+2 n_{2}^{2}\left(4 m_{2}+n_{2}\left(2 n_{2}-2\right)\right) M_{1}\left(G_{1}\right)+\left(10 m_{1} n_{2}-\right.$ $\left.4 n_{1}\right) M_{1}\left(G_{2}\right)+2 n_{2}^{2} m_{1}\left(2 n_{2}-2\right)^{2}+4 n_{1} m_{2}+16\left(n_{2}-2\right) m_{1} m_{2} n_{2}$.

Proof. Let $\left\{x_{1}, x_{2}, \ldots, x_{n_{1}}\right\}$ and $\left\{y_{1}, y_{2}, \ldots, y_{n_{2}}\right\}$ be the vertex sets of $G_{1}$ and $G_{2}$, respectively. From the definition of first reformulated Zagreb index and the structure of the graph $G_{1}\left[G_{2}\right]_{S}$, we have

$$
\begin{aligned}
E M_{1}\left(G_{1}\left[G_{2}\right]_{S}\right)= & \sum_{\left(x_{1}, y_{1}\right)\left(x_{2}, y_{2}\right) \in E\left(G_{1}\left[G_{2}\right]_{s}\right)}\left(d_{G_{1}\left[G_{2}\right]_{S}}\left(\left(x_{1}, y_{1}\right)\right)+d_{G_{1}\left[G_{2}\right]_{S}}\left(\left(x_{2}, y_{2}\right)\right)-2\right)^{2} \\
= & \sum_{x_{1}=x_{2} \in V\left(G_{1}\right)} \sum_{y_{1} y_{2} \in E\left(G_{2}\right)}\left(d_{G_{1}\left[G_{2}\right]_{S}}\left(\left(x_{1}, y_{1}\right)\right)+d_{G_{1}\left[G_{2}\right]_{S}}\left(\left(x_{2}, y_{2}\right)\right)-2\right)^{2} \\
& +\sum_{x_{1} x_{2} \in E\left(S\left(G_{1}\right)\right)} \sum_{y_{1} \in V\left(G_{2}\right)} \sum_{y_{2} \in V\left(G_{2}\right)}\left(d_{G_{1}\left[G_{2}\right]_{S}}\left(\left(x_{1}, y_{1}\right)\right)+d_{G_{1}\left[G_{2}\right]_{S}}\left(\left(x_{2}, y_{2}\right)\right)-2\right)^{2} \\
= & A_{1}+A_{2},
\end{aligned}
$$

where $A_{1}$ and $A_{2}$ are the sums of the terms, in order.

We shall calculate $A_{1}$ and $A_{2}$ of (1) separately. First we calculate the sum

$$
A_{1}=\sum_{x_{1}=x_{2} \in V\left(G_{1}\right)} \sum_{y_{1} y_{2} \in E\left(G_{2}\right)}\left(d_{G_{1}\left[G_{2}\right]_{s}}\left(\left(x_{1}, y_{1}\right)\right)+d_{G_{1}\left[G_{2}\right]_{s}}\left(\left(x_{2}, y_{2}\right)\right)-2\right)^{2} .
$$


Four new operations related to composition and ...

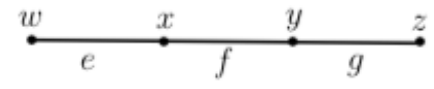

G

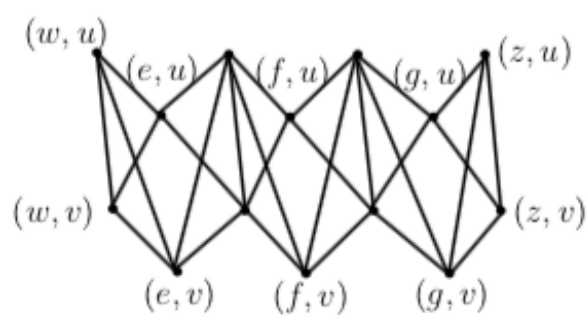

$G[H]_{S}$

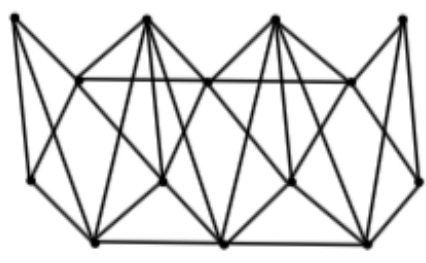

$G[H]_{Q}$
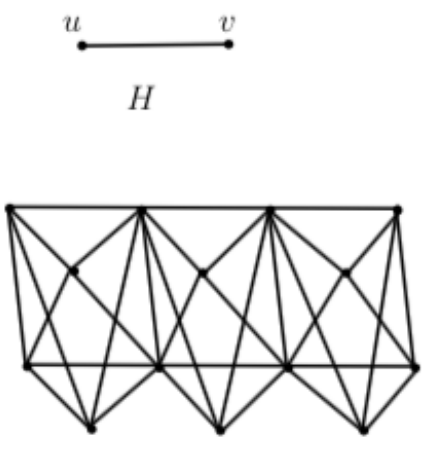

$G[H]_{R}$

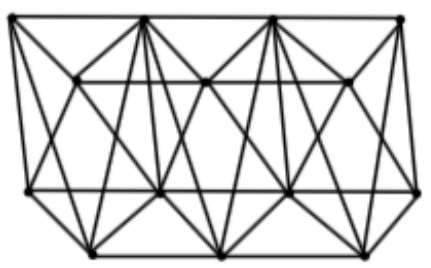

$G[H]_{T}$

Figure 3. The graph $G, H$, and its $G[H]_{T}$.

For each vertex $\left(x_{i}, y_{j}\right)$ in $G_{1}\left[G_{2}\right]_{S}$, the degree of $\left(x_{i}, y_{j}\right)$ is $n_{2} d_{G_{1}}\left(x_{i}\right)+d_{G_{2}}\left(y_{j}\right)$. Thus

$$
\begin{aligned}
A_{1} & =\sum_{x_{1} \in V\left(G_{1}\right)} \sum_{y_{1}}\left(n_{2} \in E\left(G_{2}\right)\right. \\
& =\sum_{x_{1} \in V\left(G_{1}\right)} \sum_{y_{1}}\left(x_{1} \in E\left(G_{2}\right)+d_{G_{2}}\left(y_{1}\right)+n_{2} d_{G_{1}}\left(x_{1}\right)+d_{G_{2}}\left(y_{2}\right)-2\right)^{2} \\
& =\sum_{x_{1} \in V\left(G_{1}\right)} \sum_{y_{1} d_{2} \in E\left(G_{2}\right)}\left(4 n_{2}^{2} d_{G_{1}}\left(x_{1}\right)^{2}+\left(d_{G_{2}}\left(y_{1}\right)+d_{G_{2}}\left(y_{2}\right)\right)-2\right)^{2} \\
& \left.\left.\left(d_{G_{2}}\left(y_{1}\right)+d_{G_{2}}\right)+d_{G_{2}}\left(y_{2}\right)\right)\right)^{2}+4+4 n_{2} d_{G_{1}}\left(x_{1}\right) \\
& \left.n_{2} d_{G_{1}}\left(x_{1}\right)-4\left(d_{G_{2}}\left(y_{1}\right)+d_{G_{2}}\left(y_{2}\right)\right)\right) .
\end{aligned}
$$

From the definitions of first and hyper-Zagreb indices, we obtain:

$$
A_{1}=4 n_{2}^{2} m_{2} M_{1}\left(G_{1}\right)+n_{1} H M\left(G_{2}\right)+\left(8 n_{2} m_{1}-4 n_{1}\right) M_{1}\left(G_{2}\right)+4 n_{1} m_{2}-16 n_{2} m_{1} m_{2} \text {. }
$$


Next we find the value of the sum $A_{2}$.

$$
\begin{aligned}
A_{2}= & \sum_{x_{1} x_{2} \in E\left(S\left(G_{1}\right)\right)} \sum_{y_{1} \in V\left(G_{2}\right)} \sum_{y_{2} \in V\left(G_{2}\right)}\left(d_{G_{1}\left[G_{2}\right]_{s}}\left(\left(x_{1}, y_{1}\right)\right)+d_{G_{1}\left[G_{2}\right]_{s}}\left(\left(x_{2}, y_{2}\right)\right)-2\right)^{2} \\
= & \sum_{y_{1} \in V\left(G_{2}\right)} \sum_{y_{2} \in V\left(G_{2}\right)} \sum_{\substack{x_{1} \in V\left(G_{1}\right), e \in E\left(G_{1}\right) \\
x_{1} \text { and } e \text { are incident in } G_{1}}}\left(d\left(\left(x, y_{1}\right)\right)+d\left(\left(e, y_{2}\right)\right)-2\right)^{2} \\
= & \sum_{y_{1} \in V\left(G_{2}\right)} \sum_{y_{2} \in V\left(G_{2}\right)} \sum_{\substack{x_{1} \in V\left(G_{1}\right), e \in E\left(G_{1}\right) \\
x_{1} \text { and } e \text { are incident in } G_{1}}}\left(n_{2} d_{G_{1}}\left(x_{1}\right)+d_{G_{2}}\left(y_{1}\right)+2 n_{2}-2\right)^{2} \\
= & \sum_{y_{1} \in V\left(G_{2}\right)} \sum_{y_{2} \in V\left(G_{2}\right)} \sum_{x \in V\left(G_{1}\right)} d_{G_{1}}\left(x_{1}\right)\left(n_{2}^{2} d_{G_{1}}\left(x_{1}\right)^{2}+d_{G_{2}}\left(y_{1}\right)^{2}+\left(2 n_{2}-2\right)^{2}\right. \\
& \left.+2 n_{2} d_{G_{1}}\left(x_{1}\right) d_{G_{2}}\left(y_{1}\right)+2 n_{2}\left(2 n_{2}-2\right) d_{G_{1}}\left(x_{1}\right)+2\left(2 n_{2}-2\right) d_{G_{2}}\left(y_{1}\right)\right) .
\end{aligned}
$$

By the definitions of $F$-index and first Zagreb index, we get

$$
\begin{aligned}
A_{2}= & n_{2}^{4} F\left(G_{1}\right)+\left(4 n_{2}^{2} m_{2}+2 n_{2}^{3}\left(2 n_{2}-2\right)\right) M_{1}\left(G_{1}\right)+2 n_{2} m_{1} M_{1}\left(G_{2}\right)+2 m_{1} n_{2}^{2}\left(2 n_{2}-2\right)^{2} \\
& +8\left(2 n_{2}-2\right) n_{2} m_{1} m_{2} .
\end{aligned}
$$

Adding $A_{1}$ and $A_{2}$, we obtain the required result.

Next we obtain the first reformulated Zagreb index of the graph $G_{1}\left[G_{2}\right]_{R}$.

Theorem 2.2. Let $G_{i}$ be a graph with $n_{i}$ vertices and $m_{i}$ edges, $i=1,2$. Then $E M_{1}\left(G_{1}\left[G_{2}\right]_{R}\right)=$ $4 n_{2}^{4} H M\left(G_{1}\right)+n_{1} H M\left(G_{2}\right)+4 n_{2}^{4} F\left(G_{1}\right)+8 n_{2}^{2}\left(5 m_{2}-n_{2}\right) M_{1}\left(G_{1}\right)+\left(20 n_{2} m_{1}-4 n_{1}\right) M_{1}\left(G_{2}\right)+$ $4 m_{1} n_{2}^{2}-8 m_{1} m_{2}\left(m_{2}+8 n_{2}-2 n_{2}^{2}\right)$.

Proof. By the definition of first reformulated Zagreb index and the structure of $G_{1}\left[G_{2}\right]_{R}$,

$$
\begin{aligned}
E M_{1}\left(G_{1}\left[G_{2}\right]_{R}\right)= & \sum_{\left(x_{1}, y_{1}\right)\left(x_{2}, y_{2}\right) \in E\left(G_{1}\left[G_{2}\right]_{R}\right)}\left(d_{G_{1}\left[G_{2}\right]_{R}}\left(\left(x_{1}, y_{1}\right)\right)+d_{G_{1}\left[G_{2}\right]_{R}}\left(\left(x_{2}, y_{2}\right)\right)-2\right)^{2} \\
= & \sum_{x_{1}=x_{2} \in V\left(G_{1}\right)} \sum_{y_{1} y_{2} \in E\left(G_{2}\right)}\left(d_{G_{1}\left[G_{2}\right]_{R}}\left(\left(x_{1}, y_{1}\right)\right)+d_{G_{1}\left[G_{2}\right]_{R}}\left(\left(x_{2}, y_{2}\right)\right)-2\right)^{2} \\
& +\sum_{x_{1} x_{2} \in E\left(R\left(G_{1}\right)\right)} \sum_{y_{1} \in V\left(G_{2}\right)} \sum_{y_{2} \in V\left(G_{2}\right)}\left(d_{G_{1}\left[G_{2}\right]_{R}}\left(\left(x_{1}, y_{1}\right)\right)+d_{G_{1}\left[G_{2}\right]_{R}}\left(\left(x_{2}, y_{2}\right)\right)-2\right)^{2} \\
= & A_{1}+A_{2}
\end{aligned}
$$

where $A_{1}$ and $A_{2}$ are the sums of the terms, in order.

We shall obtain the value of $A_{1}$ and $A_{2}$ of (2) separately. 


$$
\begin{aligned}
A_{1} & =\sum_{x_{1}=x_{2} \in V\left(G_{1}\right)} \sum_{y_{1} y_{2} \in E\left(G_{2}\right)}\left(d_{G_{1}\left[G_{2}\right]_{R}}\left(\left(x_{1}, y_{1}\right)\right)+d_{G_{1}\left[G_{2}\right]_{R}}\left(\left(x_{2}, y_{2}\right)\right)-2\right)^{2} \\
= & \sum_{x_{1} \in V\left(G_{1}\right)} \sum_{y_{1} y_{2} \in E\left(G_{2}\right)}\left(n_{2} d_{R\left(G_{1}\right)}\left(x_{1}\right)+d_{G_{2}}\left(y_{1}\right)+n_{2} d_{R\left(G_{1}\right)}\left(x_{1}\right)+d_{G_{2}}\left(y_{2}\right)-2\right)^{2} \\
= & \sum_{x_{1} \in V\left(G_{1}\right)} \sum_{y_{1} y_{2} \in E\left(G_{2}\right)}\left(4 n_{2} d_{G_{1}}\left(x_{1}\right)+\left(d_{G_{2}}\left(y_{1}\right)+d_{G_{2}}\left(y_{2}\right)\right)-2\right)^{2} \\
= & \sum_{x_{1} \in V\left(G_{1}\right)} \sum_{y_{1} y_{2} \in E\left(G_{2}\right)}\left(16 n_{2}^{2} d_{G_{1}}\left(x_{1}\right)^{2}+\left(d_{G_{2}}\left(y_{1}\right)+d_{G_{2}}\left(y_{2}\right)\right)^{2}+4\right. \\
& \left.+8 n_{2} d_{G_{1}}\left(x_{1}\right)\left(d_{G_{2}}\left(y_{1}\right)+d_{G_{2}}\left(y_{2}\right)\right)-16 n_{2} d_{G_{1}}\left(x_{1}\right)-4\left(d_{G_{2}}\left(y_{1}\right)+d_{G_{2}}\left(y_{2}\right)\right)\right) \\
= & 16 n_{2}^{2} m_{2} M_{1}\left(G_{1}\right)+n_{1} H M\left(G_{2}\right)+\left(16 n_{2} m_{1}-4 n_{1}\right) M_{1}\left(G_{2}\right)+4 n_{1} m_{2}-32 n_{2} m_{1} m_{2} .
\end{aligned}
$$

$$
\begin{aligned}
A_{2}= & \sum_{x_{1} x_{2} \in E\left(R\left(G_{1}\right)\right)} \sum_{\substack{y_{1} \in V\left(G_{2}\right) \\
y_{2} \in V\left(G_{2}\right)}}\left(d_{G_{1}\left[G_{2}\right]_{R}}\left(\left(x_{1}, y_{1}\right)\right)+d_{G_{1}\left[G_{2}\right]_{R}}\left(\left(x_{2}, y_{2}\right)\right)-2\right)^{2} \\
= & \sum_{y_{1} \in V\left(G_{2}\right)} \sum_{y_{2} \in V\left(G_{2}\right)} \sum_{x_{1} x_{2} \in V\left(G_{1}\right)}\left(d\left(\left(x_{1}, y_{1}\right)\right)+d\left(\left(x_{2}, y_{2}\right)\right)-2\right)^{2} \\
& +\sum_{y_{1} \in V\left(G_{2}\right)} \sum_{y_{2} \in V\left(G_{2}\right)} \sum_{\substack{\left.x_{1} \in V\left(G_{1}\right), x_{2} \in V\left(R\left(G_{1}\right)\right) \\
x_{1} \in\left(G_{1}\right)\right)-V\left(G_{1}\right)}}\left(d\left(x_{1}, y_{1}\right)+d\left(x_{2}, y_{2}\right)-2\right)^{2} \\
= & A_{2}^{\prime}+A_{2}^{\prime \prime},
\end{aligned}
$$

where

$$
\begin{aligned}
A_{2}^{\prime}= & \left.\sum_{y_{1} \in V\left(G_{2}\right)} \sum_{y_{2} \in V\left(G_{2}\right)} \sum_{x_{1} x_{2} \in V\left(G_{1}\right)}\left(d\left(x_{1}, y_{1}\right)+d\left(x_{2}, y_{2}\right)\right)-2\right)^{2} \\
= & \sum_{y_{1} \in V\left(G_{2}\right)} \sum_{y_{2} \in V\left(G_{2}\right)} \sum_{x_{1} x_{2} \in V\left(G_{1}\right)}\left(n_{2} d_{R\left(G_{1}\right)}\left(x_{1}\right)+d_{G_{2}}\left(y_{1}\right)+n_{2} d_{R\left(G_{1}\right)}\left(x_{2}\right)+d_{G_{2}}\left(y_{2}\right)-2\right)^{2} \\
= & \sum_{y_{1} \in V\left(G_{2}\right)} \sum_{y_{2} \in V\left(G_{2}\right)} \sum_{x_{1} x_{2} \in V\left(G_{1}\right)}\left(2 n_{2} d_{G_{1}}\left(x_{1}\right)+d_{G_{2}}\left(y_{1}\right)+2 n_{2} d_{G_{1}}\left(x_{2}\right)+d_{G_{2}}\left(y_{2}\right)-2\right)^{2} \\
= & \sum_{y_{1} \in V\left(G_{2}\right)} \sum_{y_{2} \in V\left(G_{2}\right)} \sum_{x_{1} x_{2} \in V\left(G_{1}\right)}\left(4 n_{2}^{2}\left(d_{G_{1}}\left(x_{1}\right)+d_{G_{1}}\left(x_{2}\right)\right)^{2}+d_{G_{2}}\left(y_{1}\right)^{2}+d_{G_{2}}\left(y_{2}\right)^{2}+4\right. \\
& +2 d_{G_{2}}\left(y_{1}\right) d_{G_{2}}\left(y_{2}\right)-4 d_{G_{2}}\left(y_{1}\right)-4 d_{G_{2}}\left(y_{2}\right)+\left(4 n_{2} d_{G_{2}}\left(y_{1}\right)+4 n_{2} d_{G_{2}}\left(y_{2}\right)-8 n_{2}\right) \\
& \left.\left(d_{G_{1}}\left(x_{1}\right)+d_{G_{1}}\left(x_{2}\right)\right)\right) \\
= & 4 n_{2}^{4} H M\left(G_{1}\right)+2 m_{1} n_{2} M_{1}\left(G_{2}\right)+4 n_{2}\left(4 m_{2} n_{2}-2 n_{2}^{2}\right) M_{1}\left(G_{1}\right)+m_{1}\left(4 n_{2}^{2}-8 m_{2}^{2}-16 n_{2} m_{2}\right) .
\end{aligned}
$$


and

$$
\begin{aligned}
& A_{2}^{\prime \prime}=\sum_{y_{1} \in V\left(G_{2}\right)} \sum_{y_{2} \in V\left(G_{2}\right)} \sum_{\substack{x_{1} x_{2} \in E\left(R\left(G_{1}\right)\right) \\
x_{1} \in V\left(G_{1}\right), x_{2} \in V\left(R\left(G_{1}\right)\right)-V\left(G_{1}\right)}}\left(d\left(x_{1}, y_{1}\right)+d\left(x_{2}, y_{2}\right)-2\right)^{2} \\
& =\sum_{y_{1} \in V\left(G_{2}\right)} \sum_{y_{2} \in V\left(G_{2}\right)} \sum_{\substack{x_{1} x_{2} \in E\left(R\left(G_{1}\right)\right) \\
x_{1} \in V\left(G_{1}\right), x_{2} \in V\left(R\left(G_{1}\right)\right)-V\left(G_{1}\right)}}\left(n_{2} d_{R\left(G_{1}\right)}\left(x_{1}\right)+d_{G_{2}}\left(y_{1}\right)+n_{2} d_{R\left(G_{1}\right)}\left(x_{2}\right)-2\right)^{2} \\
& =\sum_{y_{1} \in V\left(G_{2}\right)} \sum_{y_{2} \in V\left(G_{2}\right)} \sum_{\substack{x_{1} x_{2} \in E\left(R\left(G_{1}\right)\right) \\
x_{1} \in V\left(G_{1}\right), x_{2} \in V\left(R\left(G_{1}\right)\right)-V\left(G_{1}\right)}}\left(2 n_{2} d_{G_{1}}\left(x_{1}\right)+d_{G_{2}}\left(y_{1}\right)+2 n_{2}-2\right)^{2} \\
& =\sum_{y_{1} \in V\left(G_{2}\right)} \sum_{y_{2} \in V\left(G_{2}\right)} \sum_{x_{1} \in V\left(G_{1}\right)} d_{G_{1}}\left(x_{1}\right)\left(4 n_{2}^{2} d_{G_{1}}\left(x_{1}\right)^{2}+d_{G_{2}}\left(y_{1}\right)^{2}+\left(2 n_{2}-2\right)^{2}\right. \\
& \left.+4 n_{2} d_{G_{1}}\left(x_{1}\right) d_{G_{2}}\left(y_{1}\right)+4 n_{2}\left(2 n_{2}-2\right) d_{G_{1}}\left(x_{1}\right)+2\left(2 n_{2}-2\right) d_{G_{2}}\left(y_{1}\right)\right) \\
& =4 n_{2}^{4} F\left(G_{1}\right)+2 m_{1} n_{2} M_{1}\left(G_{2}\right)+8\left(2 n_{2}-2\right) n_{2} m_{1} m_{2}+\left(8 n_{2}^{2} m_{2}+4 n_{2}^{3}\left(2 n_{2}-2\right)\right) M_{1}\left(G_{1}\right) \text {. }
\end{aligned}
$$

From $A_{2}^{\prime}$ and $A_{2}^{\prime \prime}$, we have $A_{2}=4 n_{2}^{4} H M\left(G_{1}\right)+4 n_{2}^{2} F\left(G_{1}\right)+4 m_{1} n_{2} M_{1}\left(G_{2}\right)+8 n_{2}^{2}\left(3 m_{2}-\right.$ $\left.n_{2}\right) M_{1}\left(G_{1}\right)+4 n_{2}^{2} m_{1}-8 m_{1} m_{2}\left(m_{2}+4 n_{2}-2 n_{2}^{2}\right)$.

Using (2) and the sums $A_{1}, A_{2}$ we obtain the desired result.

Next we find the first reformulated Zagreb index of $G_{1}\left[G_{2}\right]_{Q}$.

Theorem 2.3. Let $G_{i}$ be a graph with $n_{i}$ vertices and $m_{i}$ edges, $i=1,2$. Then $E M_{1}\left(G_{1}\left[G_{2}\right]_{Q}\right)=$ $2 n_{2}^{4} H M\left(G_{1}\right)+n_{1} H M\left(G_{2}\right)+3 n_{2}^{4} F\left(G_{1}\right)+n_{2}^{2}\left(n_{2}^{2} H M\left(L\left(G_{1}\right)\right)+2 n_{2}\left(4 n_{2}-2\right) M_{1}\left(L\left(G_{1}\right)\right)\right)+$ $n_{2}^{2}\left(16 m_{2}-8 n_{2}-2\right) M_{1}\left(G_{1}\right)+\left(10 n_{2} m_{1}-4 n_{1}\right) M_{1}\left(G_{2}\right)+4 n_{2}^{4} M_{2}\left(G_{1}\right)+4 n_{1} m_{2}+8 n_{2} m_{1}\left(n_{2}-\right.$ $\left.4 m_{2}\right)-n_{2}^{2} m_{1}\left(4 n_{2}-2\right)^{2}$.

Proof. By the definition of first reformulated Zagreb index,

$$
\begin{aligned}
E M_{1}\left(G_{1}\left[G_{2}\right]_{Q}\right)= & \sum_{\left(x_{1}, y_{1}\right)\left(x_{2}, y_{2}\right) \in E\left(G_{1}\left[G_{2}\right]_{Q}\right)}\left(d_{G_{1}\left[G_{2}\right]_{R}}\left(\left(x_{1}, y_{1}\right)\right)+d_{G_{1}\left[G_{2}\right]_{Q}}\left(\left(x_{2}, y_{2}\right)\right)-2\right)^{2} \\
= & \sum_{x_{1}=x_{2} \in V\left(G_{1}\right)} \sum_{y_{1} y_{2} \in E\left(G_{2}\right)}\left(d_{G_{1}\left[G_{2}\right]_{Q}}\left(\left(x_{1}, y_{1}\right)\right)+d_{G_{1}\left[G_{2}\right]_{Q}}\left(\left(x_{2}, y_{2}\right)\right)-2\right)^{2} \\
& +\sum_{x_{1} x_{2} \in E\left(Q\left(G_{1}\right)\right)} \sum_{y=V\left(G_{2}\right)} \sum_{y_{2} \in V\left(G_{2}\right)}\left(d_{G_{1}\left[G_{2}\right]_{Q}}\left(\left(x_{1}, y_{1}\right)\right)+d_{G_{1}\left[G_{2}\right]_{Q}}\left(\left(x_{2}, y_{2}\right)\right)-2\right)^{2} \\
= & A_{1}+A_{2},
\end{aligned}
$$

where $A_{1}$ and $A_{2}$ are the sums of the terms, in order. 
We shall calculate $A_{1}$ and $A_{2}$ of 4 separately.

$$
\begin{aligned}
& A_{1}=\sum_{x_{1}=x_{2} \in V\left(G_{1}\right)} \sum_{y_{1} y_{2} \in E\left(G_{2}\right)}\left(d_{G_{1}\left[G_{2}\right]_{Q}}\left(\left(x_{1}, y_{1}\right)\right)+d_{G_{1}\left[G_{2}\right]_{Q}}\left(\left(x_{2}, y_{2}\right)\right)-2\right)^{2}
\end{aligned}
$$

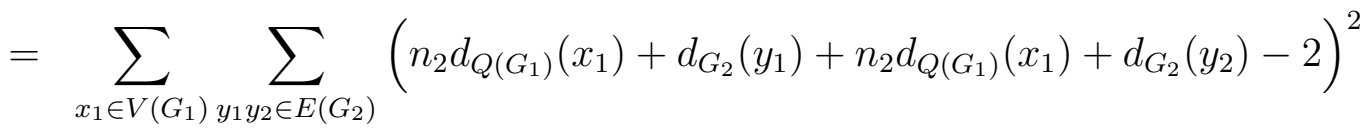

$$
\begin{aligned}
& =\sum_{x_{1} \in V\left(G_{1}\right)} \sum_{y_{1}}\left(2 n_{2} d_{G_{1}}\left(x_{1}\right)+\left(d_{G_{2}}\left(y_{1}\right)+d_{G_{2}}\left(y_{2}\right)\right)-2\right)^{2} \\
& =\sum_{x_{1} \in V\left(G_{1}\right)} \sum_{y_{1}, y_{2} \in E\left(G_{2}\right)}\left(4 n_{2}^{2} d_{G_{1}}\left(x_{1}\right)^{2}+\left(d_{G_{2}}\left(y_{1}\right)+d_{G_{2}}\left(y_{2}\right)\right)^{2}+4\right. \\
& \left.+4 n_{2} d_{G_{1}}\left(x_{1}\right)\left(d_{G_{2}}\left(y_{1}\right)+d_{G_{2}}\left(y_{2}\right)\right)-8 n_{2} d_{G_{1}}\left(x_{1}\right)-4\left(d_{G_{2}}\left(y_{1}\right)+d_{G_{2}}\left(y_{2}\right)\right)\right) \\
& =4 n_{2}^{2} m_{2} M_{1}\left(G_{1}\right)+n_{1} H M\left(G_{2}\right)+\left(8 n_{2} m_{1}-4 n_{1}\right) M_{1}\left(G_{2}\right)+4 n_{1} m_{2}-16 n_{2} m_{1} m_{2} \text {. } \\
& A_{2}=\sum_{x_{1} x_{2} \in E\left(Q\left(G_{1}\right)\right)} \sum_{y_{1} \in V\left(G_{2}\right)} \sum_{y_{2} \in V\left(G_{2}\right)}\left(d_{G_{1}\left[G_{2}\right]_{Q}}\left(\left(x_{1}, y_{1}\right)\right)+d_{G_{1}\left[G_{2}\right]_{Q}}\left(\left(x_{2}, y_{2}\right)\right)-2\right)^{2} \\
& \left.=\sum_{y_{1} \in V\left(G_{2}\right)} \sum_{y_{2} \in V\left(G_{2}\right)} \sum_{\substack{x_{1} x_{2} \in E\left(Q\left(G_{1}\right)\right) \\
x_{1} \in V\left(G_{1}\right), x_{2} \in V\left(Q\left(G_{1}\right)\right)-V\left(G_{1}\right)}}\left(d\left(x_{1}, y_{1}\right)+d\left(x_{2}, y_{2}\right)\right)-2\right)^{2} \\
& +\sum_{y_{1} \in V\left(G_{2}\right)} \sum_{y_{2} \in V\left(G_{2}\right)} \sum_{\substack{x_{1} x_{2} \in E\left(Q\left(G_{1}\right)\right) \\
x_{1}, x_{2} \in V\left(Q\left(G_{1}\right)\right)-V\left(G_{1}\right)}}\left(d\left(x_{1}, y_{1}\right)+d\left(x_{2}, y_{2}\right)-2\right)^{2} \\
& =A_{2}^{\prime}+A_{2}^{\prime \prime}
\end{aligned}
$$


where

$$
\begin{aligned}
& A_{2}^{\prime}=\sum_{y_{1} \in V\left(G_{2}\right)} \sum_{y_{2} \in V\left(G_{2}\right)} \sum_{\substack{x_{1} x_{2} \in E\left(Q\left(G_{1}\right)\right) \\
x_{1} \in V\left(G_{1}\right), x_{2} \in V\left(Q\left(G_{1}\right)\right)-V\left(G_{1}\right)}}\left(d\left(\left(x_{1}, y_{1}\right)\right)+d\left(\left(x_{2}, y_{2}\right)\right)-2\right)^{2} \\
& =\sum_{y_{1} \in V\left(G_{2}\right)} \sum_{y_{2} \in V\left(G_{2}\right)} \sum_{\substack{x_{1} x_{2} \in E\left(Q\left(G_{1}\right)\right) \\
x_{1} \in V\left(G_{1}\right), x_{2} \in V\left(Q\left(G_{1}\right)\right)-V\left(G_{1}\right)}}\left(n_{2} d_{Q\left(G_{1}\right)}\left(x_{1}\right)+d_{G_{2}}\left(y_{1}\right)+n_{2} d_{Q\left(G_{1}\right)}\left(x_{2}\right)-2\right)^{2} \\
& =\sum_{y_{1} \in V\left(G_{2}\right)} \sum_{y_{2} \in V\left(G_{2}\right)} \sum_{x_{1} x_{2} \in E\left(Q\left(G_{1}\right)\right)}\left(n_{2} d_{G_{1}}\left(x_{1}\right)+d_{G_{2}}\left(y_{1}\right)+n_{2} d_{Q\left(G_{1}\right)}\left(x_{2}\right)-2\right)^{2} \\
& x_{1} \in V\left(G_{1}\right), x_{2} \in V\left(Q\left(G_{1}\right)\right)-V\left(G_{1}\right) \\
& =\sum_{y_{1} \in V\left(G_{2}\right)} \sum_{y_{2} \in V\left(G_{2}\right)} \sum_{x_{1} x_{2} \in E\left(Q\left(G_{1}\right)\right)}\left(n_{2}^{2} d_{G_{1}}\left(x_{1}\right)^{2}+\left(d_{G_{2}}\left(y_{1}\right)-2\right)^{2}+n_{2}^{2} d_{Q\left(G_{1}\right)}\left(x_{2}\right)^{2}\right. \\
& x_{1} \in V\left(G_{1}\right), x_{2} \in V\left(Q\left(G_{1}\right)\right)-V\left(G_{1}\right) \\
& \left.+2 n_{2} d_{G_{1}}\left(x_{1}\right)\left(d_{G_{2}}\left(y_{1}\right)-2\right)+2 n_{2}^{2} d_{G_{1}}\left(x_{1}\right) d_{Q\left(G_{1}\right)}\left(x_{2}\right)+2 n_{2}\left(d_{G_{2}}\left(y_{1}\right)-2\right) d_{Q\left(G_{1}\right)}\left(x_{2}\right)\right) \\
& =n_{2}^{4} F\left(G_{1}\right)+2 n_{2}^{2}\left(2 m_{2}-2 n_{2}\right) M_{1}\left(G_{1}\right)+2 n_{2} m_{1}\left(M_{1}\left(G_{2}\right)+4 n_{2}-8 m_{2}\right) \\
& +\sum_{y_{1} \in V\left(G_{2}\right)} \sum_{y_{2} \in V\left(G_{2}\right)} \sum_{x_{1} x_{2} \in E\left(Q\left(G_{1}\right)\right)}\left(n_{2}^{2} d_{Q\left(G_{1}\right)}\left(x_{2}\right)^{2}+\left(2 n_{2}^{2} d_{G_{1}}\left(x_{1}\right)\right.\right. \\
& x_{1} \in V\left(G_{1}\right), x_{2} \in V\left(Q\left(G_{1}\right)\right)-V\left(G_{1}\right) \\
& \left.\left.+2 n_{2}\left(d_{G_{2}}\left(y_{1}\right)-2\right)\right) d_{Q\left(G_{1}\right)}\left(x_{2}\right)\right)
\end{aligned}
$$

One can see that for a vertex $x_{2} \in V\left(Q\left(G_{1}\right)\right)-V\left(G_{1}\right), d_{Q\left(G_{1}\right)}\left(x_{2}\right)=d_{G_{1}}(x)+d_{G_{1}}(w)$, where $x_{2}=x w \in E\left(G_{1}\right)$. Thus

$$
\begin{aligned}
A_{2}^{\prime}= & n_{2}^{4} F\left(G_{1}\right)+2 n_{2}^{2}\left(2 m_{2}-2 n_{2}\right) M_{1}\left(G_{1}\right)+2 n_{2} m_{1}\left(M_{1}\left(G_{2}\right)+4 n_{2}-8 m_{2}\right) \\
& +\sum_{y_{1} \in V\left(G_{2}\right)} \sum_{y_{2} \in V\left(G_{2}\right)}\left(n_{2}^{2}\left(d_{G_{1}}(x)+d_{G_{1}}(w)\right)^{2}\right. \\
& \left.+2 n_{2}^{2} d_{G_{1} \in V\left(G_{1}\right), x_{2} \in V\left(x_{1} \in\left(G_{1}\right)\right)}\left(d_{G_{1}}(x)+d_{G_{1}}(w)\right)+2 n_{2}\left(d_{G_{2}}\left(y_{1}\right)-2\right)\left(d_{G_{1}}(x)+d_{G_{1}}(w)\right)\right) \\
= & n_{2}^{4} F\left(G_{1}\right)+2 n_{2}^{2}\left(2 m_{2}-2 n_{2}\right) M_{1}\left(G_{1}\right)+2 n_{2} m_{1}\left(M_{1}\left(G_{2}\right)+4 n_{2}-8 m_{2}\right) \\
& +2 n_{2}^{4} H M\left(G_{1}\right)+2 n_{2}^{4}\left(F\left(G_{1}\right)+2 M_{2}\left(G_{1}\right)\right)+4 n_{2}^{2}\left(2 m_{2}-2 n_{2}\right) M_{1}\left(G_{1}\right) \\
= & 2 n_{2}^{4} H M\left(G_{1}\right)+3 n_{2}^{4} F\left(G_{1}\right)+12 n_{2}^{2}\left(m_{2}-n_{2}\right) M_{1}\left(G_{1}\right)+2 n_{2} m_{1} M_{1}\left(G_{2}\right) \\
& +4 n_{2}^{4} M_{2}\left(G_{1}\right)+2 m_{1} n_{2}\left(4 n_{2}-8 m_{2}\right) .
\end{aligned}
$$




$$
\begin{aligned}
A_{2}^{\prime \prime} & =\sum_{y_{1} \in V\left(G_{2}\right)} \sum_{y_{2} \in V\left(G_{2}\right)} \sum_{\substack{x_{1} x_{2} \in E\left(Q\left(G_{1}\right)\right) \\
x_{1}, x_{2} \in V\left(Q\left(G_{1}\right)\right)-V\left(G_{1}\right)}}\left(d\left(x_{1}, y_{1}\right)+d\left(x_{2}, y_{2}\right)-2\right)^{2} \\
& =\sum_{y_{1} \in V\left(G_{2}\right)} \sum_{y_{2} \in V\left(G_{2}\right)} \sum_{\substack{x_{1} \in E\left(Q\left(G_{1}\right)\right) \\
x_{1}, x_{2} \in V\left(Q\left(G_{1}\right)\right)-V\left(G_{1}\right)}}\left(n_{2} d_{Q\left(G_{1}\right)}\left(x_{1}\right)+n_{2} d_{Q\left(G_{1}\right)}\left(x_{2}\right)-2\right)^{2} \\
& =n_{2}^{2} \sum_{w_{i} w_{j}, w_{j} w_{k} \in E\left(G_{1}\right)}\left(n_{2}\left(d_{G_{1}}\left(w_{i}\right)+d_{G_{1}}\left(w_{j}\right)\right)+n_{2}\left(d_{G_{1}}\left(w_{j}\right)+d_{G_{1}}\left(w_{k}\right)\right)-2\right)^{2} \\
& =n_{2}^{2} \sum_{X_{i} X_{j} \in E\left(L\left(G_{1}\right)\right)}\left(n_{2} d_{L\left(G_{1}\right)}\left(X_{i}\right)+n_{2} d_{L\left(G_{1}\right)}\left(X_{j}\right)+4 n_{2}-2\right)^{2},
\end{aligned}
$$

where $X_{i}$ and $X_{j}$ are vertices of $L\left(G_{1}\right)$

$$
\begin{aligned}
= & n_{2}^{2} \sum_{X_{i} X_{j} \in E\left(L\left(G_{1}\right)\right)}\left(n_{2}^{2}\left(d_{L\left(G_{1}\right)}\left(X_{i}\right)+d_{L\left(G_{1}\right)}\left(X_{j}\right)\right)^{2}+\left(4 n_{2}-2\right)^{2}\right. \\
& \left.+2 n_{2}\left(4 n_{2}-2\right)\left(d_{L\left(G_{1}\right)}\left(X_{i}\right)+d_{L\left(G_{1}\right)}\left(X_{j}\right)\right)\right) \\
= & n_{2}^{2}\left(n_{2}^{2} H M\left(L\left(G_{1}\right)\right)+2\left(4 n_{2}-2\right) n_{2} M_{1}\left(L\left(G_{1}\right)\right)+\left(4 n_{2}-2\right)^{2}\left(\frac{M_{1}\left(G_{1}\right)}{2}-m_{1}\right)\right) .
\end{aligned}
$$

From the sums $A_{2}^{\prime}$ and $A_{2}^{\prime \prime}$, we have $A_{2}=2 n_{2}^{4} H M\left(G_{1}\right)+3 n_{2}^{4} F\left(G_{1}\right)+n_{2}^{2}\left(12 m_{2}-8 n_{2}-2\right) M_{1}\left(G_{1}\right)+$ $2 n_{2} m_{1} M_{1}\left(G_{2}\right)+4 n_{2}^{4} M_{2}\left(G_{1}\right)+n_{2}^{2}\left(n_{2}^{2} H M\left(L\left(G_{1}\right)\right)+2 n_{2}\left(4 n_{2}-2\right) M_{1}\left(L\left(G_{1}\right)\right)\right)+2 m_{1} n_{2}\left(4 n_{2}-\right.$ $\left.8 m_{2}\right)-n_{2}^{2} m_{1}\left(4 n_{2}-2\right)^{2}$.

Adding $A_{1}$ and $A_{2}$, we get the desired result.

Finally, we obtain the first formulated Zagreb index of $G_{1}\left[G_{2}\right]_{T}$.

Theorem 2.4. Let $G_{i}$ be a graph with $n_{i}$ vertices and $m_{i}$ edges, $i=1,2$. Then $E M_{1}\left(G_{1}\left[G_{2}\right]_{T}\right)=$ $10 n_{2}^{2} H M\left(G_{1}\right)+n_{1} H M\left(G_{2}\right)+4 n_{2}^{2} F\left(G_{1}\right)+n_{2}^{4} H M\left(L\left(G_{1}\right)\right)+2 n_{2}^{3}\left(4 n_{2}-2\right) M_{1}\left(L\left(G_{1}\right)\right)+\left(32 n_{2}^{2} m_{2}+\right.$ $\left.16 n_{2} m_{2}-16 n_{2}^{2}-8 n_{2}^{3}+2\left(2 n_{2}-1\right)^{2}\right) M_{1}\left(G_{1}\right)+\left(20 m_{1} n_{2}-4 n_{1}\right) M_{1}\left(G_{2}\right)+4 n_{1} m_{2}-8 m_{1} m_{2}\left(8 n_{1}+\right.$ $\left.m_{2}\right)-n_{2}^{2} m_{1}\left(\left(4 n_{2}-2\right)^{2}-12\right)$. 
Proof. By the definition of first formulated Zagreb index,

$$
\begin{aligned}
E M_{1}\left(G_{1}\left[G_{2}\right]_{T}\right)= & \sum_{\left(x_{1}, y_{1}\right)\left(x_{2}, y_{2}\right) \in E\left(G_{1}\left[G_{2}\right]_{T}\right)}\left(d_{G_{1}\left[G_{2}\right]_{T}}\left(\left(x_{1}, y_{1}\right)\right)+d_{G_{1}\left[G_{2}\right]_{T}}\left(\left(x_{2}, y_{2}\right)\right)-2\right)^{2} \\
= & \sum_{x_{1}=x_{2} \in V\left(G_{1}\right)} \sum_{y_{1} y_{2} \in E\left(G_{2}\right)}\left(d_{G_{1}\left[G_{2}\right]_{T}}\left(\left(x_{1}, y_{1}\right)\right)+d_{G_{1}\left[G_{2}\right]_{T}}\left(\left(x_{2}, y_{2}\right)\right)-2\right)^{2} \\
& +\sum_{x_{1} x_{2} \in E\left(T\left(G_{1}\right)\right)} \sum_{y_{1} \in V\left(G_{2}\right)} \sum_{y_{2} \in V\left(G_{2}\right)}\left(d_{G_{1}\left[G_{2}\right]_{T}}\left(\left(x_{1}, y_{1}\right)\right)+d_{G_{1}\left[G_{2}\right]_{T}}\left(\left(x_{2}, y_{2}\right)\right)-2\right)^{2} \\
= & \sum_{\substack{x_{1}=\\
x_{2} \in V\left(G_{1}\right)}} \sum_{y_{1} y_{2} \in E\left(G_{2}\right)}\left(d_{G_{1}\left[G_{2}\right]_{T}}\left(\left(x_{1}, y_{1}\right)\right)+d_{G_{1}\left[G_{2}\right]_{T}}\left(\left(x_{2}, y_{2}\right)\right)-2\right)^{2} \\
& \sum_{y_{1} \in V\left(G_{2}\right)} \sum_{y_{2} \in V\left(G_{2}\right)}\left(\sum_{x_{1} x_{2} \in E\left(G_{1}\right)}+\sum_{\left.x_{1} \in V\left(G_{1}\right), x_{2} \in V\left(T\left(G_{1}\right)\right)\right)-V\left(G_{1}\right)}\right. \\
& +\sum_{\substack{x_{1} x_{2} \in E\left(T\left(G_{1}\right)\right) \\
x_{1}, x_{2} \in V\left(T\left(G_{1}\right)\right)-V\left(G_{1}\right)}} \sum_{A_{1}+A_{2}+A_{3}+A_{4},}^{2}\left(d_{G_{1}\left[G_{2}\right]_{T}}\left(\left(x_{1}, y_{1}\right)\right)+d_{G_{1}\left[G_{2}\right]_{T}}\left(\left(x_{2}, y_{2}\right)\right)-2\right)^{2}
\end{aligned}
$$

where $A_{1}$ to $A_{4}$ are the sums of the terms, in order.

We shall calculate $A_{1}$ to $A_{4}$ of 5 separately. A similar arguments of $A_{1}$ and $A_{2}^{\prime}$ in Theorem 2.2, we have

$$
\begin{aligned}
A_{1} & =\sum_{x_{1}=x_{2} \in V\left(G_{1}\right)} \sum_{y_{1} \in y_{2} \in E\left(G_{2}\right)}\left(d_{G_{1}\left[G_{2}\right]_{T}}\left(\left(x_{1}, y_{1}\right)\right)+d_{G_{1}\left[G_{2}\right]_{T}}\left(\left(x_{2}, y_{2}\right)\right)-2\right)^{2} \\
& =16 n_{2}^{2} m_{2} M_{1}\left(G_{1}\right)+n_{1} H M\left(G_{2}\right)+\left(16 n_{2} m_{1}-4 n_{1}\right) M_{1}\left(G_{2}\right)+4 n_{1} m_{2}-32 n_{2} m_{1} m_{2} .
\end{aligned}
$$

and

$$
\begin{aligned}
A_{2} & =\sum_{y_{1} \in V\left(G_{2}\right)} \sum_{y_{2} \in E\left(G_{2}\right)} \sum_{x_{1} x_{2} \in E\left(G_{1}\right)}\left(d_{G_{1}\left[G_{2}\right]_{T}}\left(\left(x_{1}, y_{1}\right)\right)+d_{G_{1}\left[G_{2}\right]_{T}}\left(\left(x_{2}, y_{2}\right)\right)-2\right)^{2} \\
& =4 n_{2}^{4} H M\left(G_{1}\right)+2 m_{1} n_{2} M_{1}\left(G_{2}\right)+4 n_{2}\left(4 m_{2} n_{2}-2 n_{2}^{2}\right) M_{1}\left(G_{1}\right)+m_{1}\left(4 n_{2}^{2}-8 m_{2}^{2}-16 n_{2} m_{2}\right) .
\end{aligned}
$$




$$
\begin{aligned}
& A_{3}=\sum_{y_{1} \in V\left(G_{2}\right)} \sum_{y_{2} \in E\left(G_{2}\right)} \sum_{\substack{x_{1} x_{2} \in E\left(T\left(G_{1}\right)\right) \\
x_{1} \in V\left(G_{1}\right), x_{2} \in V\left(T\left(G_{1}\right)\right)-V\left(G_{1}\right)}}\left(d_{G_{1}\left[G_{2}\right]_{T}}\left(\left(x_{1}, y_{1}\right)\right)+d_{G_{1}\left[G_{2}\right]_{T}}\left(\left(x_{2}, y_{2}\right)\right)-2\right)^{2} \\
& =\sum_{y_{1} \in V\left(G_{2}\right)} \sum_{y_{2} \in E\left(G_{2}\right)} \sum_{\substack{x_{1} x_{2} \in E\left(T\left(G_{1}\right)\right) \\
x_{1} \in V\left(G_{1}\right), x_{2} \in V\left(T\left(G_{1}\right)\right)-V\left(G_{1}\right)}}\left(d_{T\left(G_{1}\right)}\left(x_{1}\right)+d_{G_{2}}\left(y_{2}\right)+d_{T\left(G_{1}\right)}\left(x_{2}\right)-2\right)^{2} \\
& =\sum_{y_{1} \in V\left(G_{2}\right)} \sum_{y_{2} \in E\left(G_{2}\right)} \sum_{\substack{x_{1} x_{2} \in E\left(T\left(G_{1}\right)\right) \\
x_{1} \in V\left(G_{1}\right), x_{2} \in V\left(T\left(G_{1}\right)\right)-V\left(G_{1}\right)}}\left(d_{R\left(G_{1}\right)}\left(x_{1}\right)^{2}+\left(d_{G_{2}}\left(y_{2}\right)-1\right)^{2}+d_{Q\left(G_{1}\right)}\left(x_{2}\right)^{2}\right. \\
& \left.+2 d_{R\left(G_{1}\right)}\left(x_{1}\right)\left(d_{G_{2}}\left(y_{2}\right)-2\right)+2\left(d_{G_{2}}\left(y_{2}\right)-2\right) d_{Q\left(G_{1}\right)}\left(x_{2}\right)+2 d_{R\left(G_{1}\right)}\left(x_{1}\right) d_{Q\left(G_{1}\right)}\left(x_{2}\right)\right)^{2} \\
& =\sum_{y_{1} \in V\left(G_{2}\right)} \sum_{y_{2} \in E\left(G_{2}\right)} \sum_{\substack{x_{1} x_{2} \in E\left(T\left(G_{1}\right)\right) \\
x_{1} \in V\left(G_{1}\right), x_{2} \in V\left(T\left(G_{1}\right)\right)-V\left(G_{1}\right)}}\left(4 d_{G_{1}}\left(x_{1}\right)^{2}+\left(d_{G_{2}}\left(y_{2}\right)-1\right)^{2}+d_{Q\left(G_{1}\right)}\left(x_{2}\right)^{2}\right. \\
& \left.+4 d_{G_{1}}\left(x_{1}\right)\left(d_{G_{2}}\left(y_{2}\right)-1\right)+2\left(d_{G_{2}}\left(y_{2}\right)-1\right) d_{Q\left(G_{1}\right)}\left(x_{2}\right)+2 d_{R\left(G_{1}\right)}\left(x_{1}\right) d_{Q\left(G_{1}\right)}\left(x_{2}\right)\right)^{2} \\
& =4 n_{2}^{2} F\left(G_{1}\right)+2 n_{2} m_{1} M_{1}\left(G_{2}\right)+6 n_{2}^{2} H M\left(G_{1}\right)+\left(16 n_{2} m_{2}-16 n_{2}^{2}\right) M_{1}\left(G_{1}\right)+8 m_{1} n_{2}\left(n_{2}-2 m_{2}\right) \text {. }
\end{aligned}
$$

A similar argument of $A_{2}^{\prime \prime}$ in Theorem 2.3 , we obtain

$$
\begin{aligned}
A_{4} & =\sum_{y_{1} \in V\left(G_{2}\right)} \sum_{y_{2} \in V\left(G_{2}\right)} \sum_{\substack{x_{1} x_{2} \in E\left(Q\left(G_{1}\right)\right) \\
x_{1}, x_{2} \in V\left(Q\left(G_{1}\right)\right)-V\left(G_{1}\right)}}\left(d_{G_{1}\left[G_{2}\right]_{T}}\left(\left(x_{1}, y_{1}\right)\right)+d_{G_{1}\left[G_{2}\right]_{T}}\left(\left(x_{2}, y_{2}\right)\right)-2\right)^{2} \\
& =n_{2}^{2}\left(n_{2}^{2} H M\left(L\left(G_{1}\right)\right)+2 n_{2}\left(4 n_{2}-2\right) M_{1}\left(L\left(G_{1}\right)\right)+\left(4 n_{2}-2\right)^{2}\left(\frac{M_{1}\left(G_{1}\right)}{2}-m_{1}\right)\right) .
\end{aligned}
$$

Adding the sums $A_{1}$ to $A_{4}$, we get the desired result.

\section{Acknowledgement}

The authors wish to thank the anonymous referees for their careful reading and constructive comments on earlier version of this article, which resulted in better presentation of this article.

\section{References}

[1] H. Deng, D. Sarala, S.K. Ayyaswamy, S. Balachandran, The Zagreb indices of four operations on graphs, Appl. Math. Comput. 275 (2016) 422-431.

[2] M. Eliasi, B. Taeri, Four new sums of graphs and their wiener indices, Discrete Appl. Math. 157 (2009) 794-803. 
[3] D. Sarala, H. Deng, S.K. Ayyaswamy, S. Balachandran, The Zagreb indices of graphs based on four new operations related to the lexicographic product, Appl. Math. Comput. 309 (2017) 156-169.

[4] J. Devillers, A.T. Balaban, Eds., Topological indices and related descriptors in QSAR and QSPR, Gordon and Breach, Amsterdam, The Netherlands, 1999.

[5] I. Gutman, O.E. Polansky, Mathematical concepts in organic chemistry, Springer-verlag, Berlin 1986.

[6] I. Gutman, N. Trinajstić, graph theory and molecular orbits. Total $\pi$-election energy of alternant hydrocarbons, Chem. Phy. Lett. 17 (1972) 535-538.

[7] M. H. Khalifeh, H. Yousefi-Azari, A. R. Ashrafi, The first and second Zagreb indices of some graph operations, Discrete Appl. Math. 157 (2009) 804-811.

[8] L. Feng, A. Ilic, Zagreb, Harary and hyper-Wiener indices of graphs with a given matching number, Appl. Math. Lett. 23 (2010) 943-948.

[9] I. Gutman, K. C. Das, The first Zagreb index 30 years after, MATCH Commun. Math. Comput. Chem. 50 (2004) 83-92.

[10] H. Hua, S. Zhang, Relations between Zagreb coindices and some distance-based topological indices, MATCH Commun. Math.Comput. Chem. in press.

[11] G.H. Shirdel. H.Rezapour, A.M. Sayadi, The hyper-Zagreb index of graph operations, Iranian. J. Math. Chem. 4 (2013) 213-220.

[12] Gutman, B. Furtula, Z. Kovijanic Vukicevic, G. Popivoda, Zagreb indices and coindices, MATCH Commun. Math. Comput. Chem. 74 (2015) 5-16.

[13] M.R. Farahani, Computing the hyper-Zagreb index of hexagonal nanotubes, J. Chem.\& Materials Research 2 (2015) 16-18.

[14] M.R. Farahani, The hyper-Zagreb index of $T U S C_{4} C_{8}(S)$ nanotubes, Int. J. Engg.\& Tech. Research, 2 (2015) 16-18.

[15] G.H. Shirdel, H. Rezapour and A.M. Sayadi, The hyper-Zagreb index of graph operations, Iranian J. Math. Chem. 4 (2013) 213-220.

[16] B. Furtula, I. Gutman, A forgotten topological index, J. Math. Chem. 53 (2015), 1184-1190.

[17] H. Deng, D. Sarala, S.K. Ayyaswamy, S. Balachandran, The Zagreb indices of four operations on graphs, Appl. Math. Comput. 275 (2016) 422-431.

[18] S. Ghobadi, M. Ghorbaninejad, The forgotten topological index of four operations on some special graphs, Bull. Math. Sci. and Appl. 16 (2016) 89-95. 
[19] F. Falahati-Nezhad, M. Azari, Bounds on the hyper-Zagreb index, J. Appl. Math. \& Informatics 34 (2016) 319 - 330.

[20] K. Pattabiraman, M. Vijayaragavan, Hyper-Zagreb indices and its coindices of graphs, Bull. Int. Math. Virtual Institute 7 (2017) 31-41.

[21] N.De, Some bounds of reformulated Zagreb indices, Appl. Math. Sci. 101 (2012)5005-5012.

[22] A.Ilić, B. Zhou, On reformulated Zagreb indices, Discrete Appl. Math. 160 (2012) 204-209.

[23] A.Milićević, S.Nikolić, N.Trinajstić, On reformulated Zagreb indices, Mol. Divers. 8 (2004) 93-399.

[24] G. Su, L. Xiong, L. Xu, B. Ma, On the maximum and minimum first reformulated Zagreb index with connectivity of at most $k$, Filomat 25 (2011) 75-83.

[25] B. Zhou, N. Trinajstić, Some properties of the reformulated Zagreb indices, J. Math. Chem. 48 (2010) 714-719. 